\title{
On the signatures of gravitational redshift: the onset of relativistic emission lines
}

\author{
A. Müller ${ }^{1}$ and M. Wold ${ }^{2}$ \\ 1 Max-Planck-Institut für Extraterrestrische Physik, PO box 1312, 85741 Garching, Germany \\ e-mail: amueller@mpe.mpg.de \\ 2 European Southern Observatory, Karl-Schwarzschild-Strasse 2, 85748 Garching, Germany
}

Received 16 May 2006 / Accepted 9 June 2006

\section{ABSTRACT}

\begin{abstract}
Aims. We quantify the effect of gravitational redshift on emission lines to explore the transition region from the Newtonian to the Einsteinian regime. With the emitting region closer to the Kerr black hole, lines are successively subjected to a stronger gravitationally induced shift and distortion. Simulated lines are compared to broad, optical emission lines observed in Mrk 110.

Methods. We simulate relativistic emission line profiles by using Kerr ray tracing techniques. Emitting regions are assumed to be thin equatorial rings in stationary Keplerian rotation. The emission lines are characterised by a generalized Doppler factor or redshift associated with the line core.

Results. With decreasing distance from the black hole, the gravitational redshift starts to smoothly deviate from the Newtonian Doppler factor: shifts of the line cores reveal an effect at levels of 0.0015 to $60 \%$ at gravitational radii ranging from $10^{5}$ to 2 . This corresponds to fully relativistic Doppler factors of 0.999985 to 0.4048 . The intrinsic line shape distortion by strong gravity i.e. very asymmetric lines occur at radii smaller than roughly ten gravitational radii.

Conclusions. Due to the asymptotical flatness of black hole space-time, GR effects are ubiquitous and their onset can be tested observationally with sufficient spectral resolution. With a resolving power of $\sim 100000$, yielding a resolution of $\approx 0.1 \AA$ for optical and near-infrared broad emission lines like $\mathrm{H} \beta$, HeII and $\mathrm{Pa} \alpha$, the gravitational redshift can be probed out to approximately 75000 gravitational radii. In general, gravitational redshift is an important indicator of black hole mass and disk inclination as recently demonstrated by observations of optical lines in Mrk 110. Comparing our simulated lines with this observations, we independently confirm an inclination angle of 30 degrees for the accretion disk. Redshift deviations induced by black hole spin can be probed only very close to the black hole e.g. with X-ray iron lines.
\end{abstract}

Key words. black hole physics - relativity - line: profiles - galaxies: active - Galaxy: nucleus - galaxies: Seyfert

\section{Introduction}

Active galactic nuclei (AGN) such as Seyfert galaxies and quasars are powered by accreting supermassive black holes (SMBHs) following the standard model that has been developed over four decades (Lynden-Bell 1969; Lynden-Bell \& Rees 1971). The masses of SMBHs lie in the range $10^{6}$ to $10^{10} M_{\odot}$, see e.g. Netzer (2003). In the standard model, clouds moving in the gravitational potential of the black hole are photoionized by the central AGN continuum, thereby producing Doppler broadened emission lines with widths of typically $10^{3}-10^{4} \mathrm{~km} \mathrm{~s}^{-1}$ (Woltjer 1959, as pioneering studies). The region where the broad lines originate is usually referred to as the broad-line region (BLR). The scale of the BLR is believed to be $10^{15}$ to $10^{17} \mathrm{~cm}$, corresponding to $\sim 10^{3}$ to $\sim 10^{5} r_{\mathrm{g}}$ or 0.6 to 60 light days for a $10^{7} M_{\odot}$ black hole. Here the gravitational radius is defined as $r_{\mathrm{g}}=G M / c^{2}$ with Newton's constant $G$, vacuum speed of light c and black hole mass $M$. Robinson et al. (1990) have presented complex models involving spherical or disk geometries for the BLR as well as rotational and radial cloud kinematics. They studied the influence of continuum variability on line profiles and found diverse line shapes exhibiting spikes, bumps and shoulders though in a non-relativistic regime. Additional velocity components in the BLR caused by disk winds (Konigl \& Kartje 1994) and a radial component have been suggested from accretion theory and radial velocity maps of the narrow-line region (Ruiz et al. 2001). However, the detailed structure and velocity field of the BLR remain unclear (Collin et al. 2006).

The broad-line clouds respond to variations in the central photoionizing continuum as suggested by strong correlations between $\mathrm{H} \beta$ response times and non-stellar optical continuum fluxes, $\tau_{\text {cent }} \propto \sqrt{F_{\mathrm{UV}}}$ (Peterson et al. 2002). This phenomenon is exploited in reverberation mapping techniques to determine both the scale of the BLR and the black hole mass (Blandford \& McKee 1982; Peterson 1993; Kaspi et al. 2000).

The idea that gravitational redshift may influence optical lines causing line asymmetries was raised by Netzer (1977). In the Seyfert-1 galaxy Akn 120, a slight redward displacement of the $\mathrm{H} \beta$ line was reported, amounting to $\Delta z \sim 0.0013$, interpreted as the result of gravitational redshift (Peterson et al. 1985). However, such effects may also arise from attenuation of the BLR or light-travel time effects, as discussed by Peterson et al. (1985). Similar studies that assume that observed effects are a result of gravitational redshift have been done for a quasar sample where the SMBH mass of QSO $0026+129$ could be roughly estimated to be $2 \times 10^{9} M_{\odot}$ (Zheng \& Sulentic 1990); this is still the current value within a factor of 2 (Czerny et al. 2004). Recently, several BLR optical emission lines in the narrow-line Seyfert-1 galaxy Mrk 110 were investigated (see Kollatschny 2003, K03 hereafter). In that work, $\mathrm{H} \alpha, \mathrm{H} \beta, \mathrm{HeI} \lambda 5876$ and HeII $\lambda 4686$ emission lines were found to possess a systematic shift to the red, 
with higher ionization lines showing larger shifts as expected in a BLR with stratified ionization structure.

In this paper, we study the gravitational redshift over a large range of distances from the central black hole. We quantify the relativistic gravitational redshift on emission lines until GR fades beyond the current observable limit. The investigation is carried out in a very general form by discussing the observed line profile as a function of the generalized GR Doppler factor ( $g$-factor) for Kerr black holes and an arbitrary velocity field of emitters, see e.g. Müller \& Camenzind (2004, M04 hereafter). Pioneering work on relativistic spectra was performed by Cunningham (1975) using transfer functions. However, the considerations of the $g$-factors in this work were restricted to minimum and maximum values of $g$ on infinitesimally narrow and thin stationary rings. Furthermore, the distance range of interest for BLRs, $10^{3}$ to $10^{5} r_{\mathrm{g}}$, has not been investigated in detail.

Corbin (1997) studied relativistic effects on emission lines from the BLR by assuming Keplerian orbits for the emitting clouds in a Schwarzschild geometry. It was found that line profiles decrease in both, width and redward centroid shift when the line emitting region moves away from the black hole.

Our goal is to accurately quantify the effects of gravitational redshift in the vicinity of a Kerr black hole. After a very general consideration that holds for any classical black hole of arbitrary mass, a more specific treatment involving optical emission lines from BLRs is addressed. For the case study of Mrk 110, it is even demonstrated how the mass of the SMBH and the inclination of the inner disk can be determined.

\section{Method, analysis tools and model}

\subsection{Relativistic ray tracing}

In contrast to Cunningham's work, emission lines are computed by ray tracing in the Kerr geometry of rotating black holes. Light rays emitted in the vicinity of the black hole travel to the observer on null geodesics in curved space-time, and in this work the observer is assumed to be located at $r_{\text {obs }}=10^{7} r_{\mathrm{g}}$. The Kerr Black Hole Ray Tracer (KBHRT) maps emitting points in the equatorial plane of a Kerr black hole to points on the observer's screen. Spectral line fluxes are computed by numerical integration over the solid angle subtended by the screen. All relativistic effects such as gravitational redshift, beaming and lensing are included, but higher order images are not considered. The complete solver has been presented in earlier work (M04).

\subsection{Analysing relativistic emission lines}

In the following, line fluxes are discussed as a function of the $g$-factor which is defined as

$g=v_{\mathrm{obs}} / v_{\mathrm{em}}=\lambda_{\mathrm{em}} / \lambda_{\mathrm{obs}}=\frac{1}{1+z}$

where $v$ and $\lambda$ denote frequency and wavelength, respectively, and the redshift is $z$. Emitter's and observer's frame of reference are indicated by subscripts "em" and "obs". A $g$-factor of unity therefore corresponds to an unshifted line, whereas $g<1$ indicates redshifted emission and $g>1$ blueshifted emission. Note that by using the $g$-factor, the simulated line profiles are discussed very generally without specifying a particular emission line.

Analysis tools for relativistic emission lines as well as line classification schemes by morphology that were introduced by M04 (Sect. 8) are utilized here. A relativistic emission line exhibiting two Doppler peaks can be characterised by several quantities:

- $g_{\text {min }}$, minimum $g$-factor that defines the terminating energy at the red wing;

- $g_{\max }$, maximum $g$-factor that defines the terminating energy at the blue wing;

- $g_{\text {rp }}$, the $g$-factor associated with the red relic Doppler peak;

- $g_{\text {bp }}$, the $g$-factor associated with the blue beamed Doppler peak;

- DPS $=g_{\mathrm{bp}}-g_{\mathrm{rp}}$, the Doppler peak spacing in energy;

- $F_{\max }$, maximum line flux;

- $F_{\text {rp }}$, line flux associated with the red relic Doppler peak;

- $F_{\mathrm{bp}}$, line flux associated with the blue beamed Doppler peak;

- DPR $=F_{\mathrm{bp}} / F_{\mathrm{rp}}$, the flux ratio of both Doppler peaks.

The existence and strength of these parameters depend on the line shape. Low inclination angles of the emitting surface i.e. face-on situations with axial observers destroy the typical structure with two distinct Doppler peaks.

Based on relativistic emission line terminology, line morphologies can be classified as triangular, double-peaked, doublehorned, shoulder-like and bumpy shapes. Triangular and shoulder-like morphologies lack a red Doppler peak. Bumpy morphology even lacks a distinct blue beaming peak either because a very steep disk emissivity suppresses emission at large disk radii or because the line originates too close to the black hole. We want to stress here that Robinson et al. (1990) found a similar terminology for non-relativistic lines but the classification scheme for relativistic lines in M04 was established independently.

In order to be able to characterize any line profile independently of its morphology, and in order quantify the shift of the resulting line centroid, we define a new quantity, $g_{\text {core }}$ :

$g_{\text {core }}=\frac{\sum_{i} g_{i} F_{i}}{\sum_{i} F_{i}}$.

The $g_{\text {core }}$ parameter is thus associated with the line core energy, i.e. the energy associated with the flux weight of the whole line. As can be seen, it is evaluated numerically by multiplying each energy bin, $g_{i}$, with the corresponding flux in that bin, $F_{i}$, and summing over the line profile.

Gravitational redshift in the weak field regime establishes pure shifts of spectral features without changing their intrinsic shape. However, gravitational redshift in the strong field regime - strong gravity - produces remarkable distortions of spectral shapes if the rest frame feature is compared to its analogue in the observer's frame. Distortion is a key feature of relativistic spectra exhibiting very skewed and asymmetric line profiles (Fabian et al. 1989; Popovic et al. 1995; Tanaka et al. 1995). These effects are important only very close to the black hole. Here we investigate how gravitational redshift changes the mode i.e. Einsteinian gravity transmutes to Newtonian gravity for emission regions moved away from the black hole.

We also define the half-energy radius as the radius where a given $g$-factor is reduced to exactly $1 / 2$ its original value. More precisely, $R_{j}$ denotes the half-energy radius associated with $j \in\left\{g_{\text {min }}, g_{\text {max }}, g_{\text {core }}, g_{\text {rp }}, g_{\text {bp }}\right\}$. Due to Eq. (1) the observed energy of the radiation associated with the specific $g$ is exactly one half of the emitted energy in the rest frame. As a measure of strong gravity $R_{\text {grp }}$ is chosen as the radius where the $g$-factor associated with the red Doppler peak is exactly $1 / 2$. This is a suitable choice because strong gravity deforms the red line wing in an extraordinary manner. 


\subsection{Emitter model: rendering parameters and emissivity}

The aim of this paper is to study gravitational redshift effects. Therefore, it is of particular interest to avoid blueshift effects that would blur or cancel the redshift. An easy way to switch off blueward displacements is tilting the emitting region to a faceon situation: axial observers with inclination angle $i=0^{\circ}$ to the emitting area have no relative motion along the line of sight to the emitter. In this case, the radiation is only affected by gravitational redshift because no motion is directed out of the plane.

For numerical reasons an inclination angle of $i=1^{\circ}$ is chosen which is close enough to the face-on situation. Other parameters are: Kerr black hole rotating at Thorne's limit $a / M=$ 0.998 (Thorne 1974) and a prograde Keplerian velocity field of emitters, $\Omega_{\mathrm{K}}=\sqrt{M} /\left(\sqrt{r^{3}}+a \sqrt{M}\right)$. We consider here stationary thin rings with emission peaking at $R_{\text {peak }}$. This is established by rendering a disk and shifting a Gaussian radial emissivity profile over the disk

$\epsilon(r) \propto \exp \left(-\frac{\left(r-R_{\text {peak }}\right)^{2}}{\sigma_{r}^{2}}\right)$,

as introduced by M04. The parameter $\sigma_{r}$ controls the width of the Gaussian or the size of the emitting region and is chosen to be 0.2 . The peak radius, $R_{\text {peak }}$, is given in units of gravitational radius. A localized Gaussian emissivity set in this way mimics a thin and narrow luminous ring with $\simeq 1 r_{\mathrm{g}}$ distance between inner and outer edge. The Gaussian guarantees a smooth but steep decay of emission at the ring edges. Note that using $r_{\mathrm{g}}$ as a natural scale allows for applications to any classical black hole including also stellar and intermediate-mass black holes.

The technique is now straightforward: the ring is shifted from large distances in asymptotically flat space-time in the direction towards the black hole where space-time curvature becomes strong. In the present work, we assume that the radial range of interest is $2 r_{\mathrm{g}}<R_{\text {peak }}<100000 r_{\mathrm{g}}$. For each simulated emission ring we determine the line core energy $g_{\text {core }}$ by applying Eq. (2) and computing the associated line redshift, $z_{\text {core }}$, from Eq. (1). These quantities allow for quantifying the gravitational redshift as a function of distance to the rotating black hole.

Further, we use the ring emitter model as a simple model for the broad-line region in AGN. In this case, the BLR clouds are distributed in an equatorial plane and follow Keplerian orbits around the black hole as proposed elsewhere (Netzer 1977; Robinson et al. 1990; Corbin 1997). Depending on radial distance to the center and orientation angle to observer, the BLR emission is influenced by Doppler effect, gravitational redshift and beaming to different extent. We show in Sect. 4 that it is possible to fit optical data to this simple, flat Keplerian BLR model and that plausible inclination angles of the inner disk can be deduced.

\section{Gravitationally redshifted emission lines}

\subsection{Redshifted line cores}

As outlined in the previous section the line cores are computed by ray tracing and their dependence on radial distance to the black hole is analysed. Figure 1 displays the core redshifts of emission lines, $z_{\text {core }}$, as a function of $R_{\text {peak }}$ for rings inclined to $i=1^{\circ}$. The redshifted lines are dominated by gravitational redshift by construction. The filled circles show that the redshift approaches $z \rightarrow 0$, i.e. that $g \rightarrow 1$, at distances of a few thousand gravitational radii from the black hole. This is the regime of nearly flat space-time and Newtonian physics. But approaching

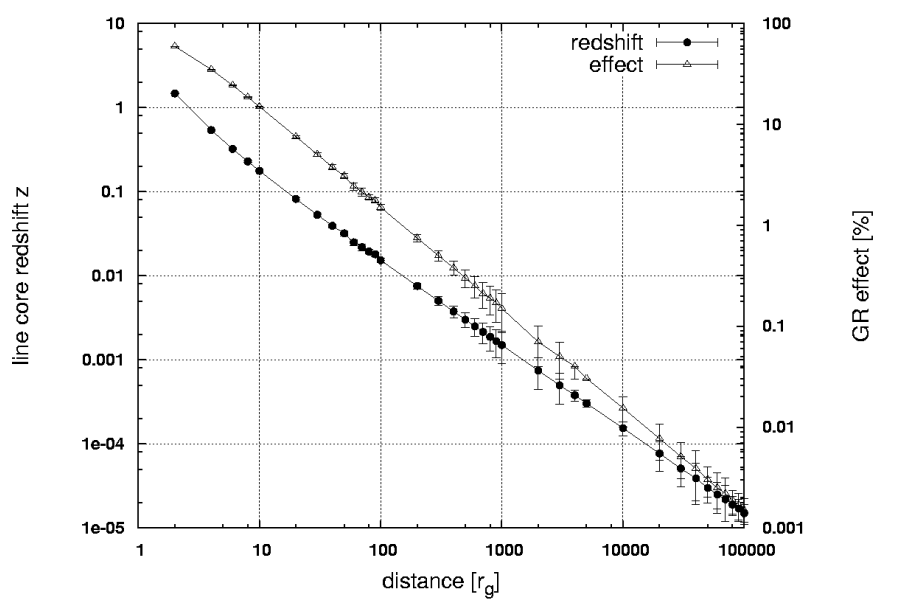

Fig. 1. Radial dependence of redshift $z$ of line cores (filled circles, left y-axis) and strength of gravitational redshift effect (triangles, right $y$-axis). Inclination angle amounts to $1^{\circ}$.

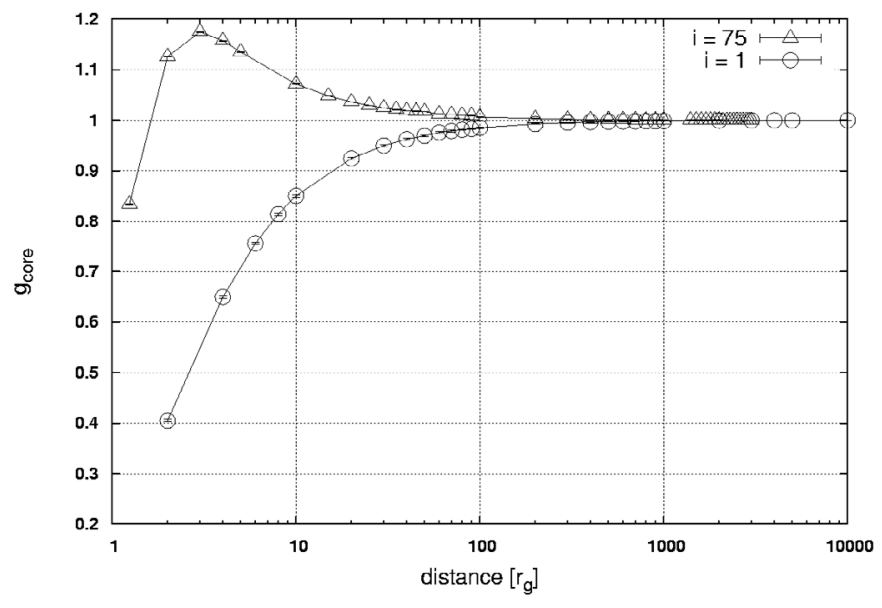

Fig. 2. Shift of line core energy in units of $g$-factor with distance to the black hole. Lowly inclined rings, $i=1^{\circ}$, are compared to highly inclined rings, $i=75^{\circ}$. Note the blueshift $g>1$ in the latter case.

the black hole, space-time curvature becomes more significant: $z$ grows rapidly and $g$ approaches zero.

The triangles illustrate the strength of the gravitational redshift effect in an alternative way. The scale of the axis on the right-hand side is computed as $\left(1-g_{\text {core }}\right) \times 100$ so that a value of $g=1$ corresponds to $0 \%$ effect and $g=0$ to a $100 \%$ effect, i.e. the gravitational redshift at the event horizon of the black hole. The error bars result from uncertainties in determining $g$-factors. Ray tracing simulations with different numerical resolutions in both disk resolution for rendering and spectral resolution for line computation yield slightly deviating values for $g_{\text {core }}$ (and other quantities in general).

Gravitational redshift can alternatively be visualised by plotting the line core energies, $g_{\text {core }}$, as a function of the peak radius for each ring. Figure 2 shows the result for $i=1^{\circ}$ and $i=75^{\circ}$. Highly inclined rings exhibit strong blueshift effects overlapping the redshift. As a consequence the core $g$-factors of the $i=75^{\circ}$ dataset never drop below 0.8 for radii larger than the marginally stable orbit. So, comparison of both orientations demonstrates that highly inclined rings are not appropriate to study gravitational redshift as pure shifting effect. It is shown later that high inclinations are well-suited for probing strong gravity. 
Table 1. Radial redshift dependence for $i=1^{\circ}$.

\begin{tabular}{cccc}
\hline \hline & & & \\
$R_{\text {peak }}\left[r_{\mathrm{g}}\right]$ & $g_{\text {core }}$ & $z_{\text {core }}$ & GR effect $[\%]$ \\
\hline 2 & 0.404763 & 1.470584 & 59.52 \\
4 & 0.649680 & 0.539220 & 35.03 \\
6 & 0.755815 & 0.323075 & 24.42 \\
8 & 0.813688 & 0.228973 & 18.63 \\
10 & 0.849776 & 0.176780 & 15.02 \\
\hline 20 & 0.924283 & 0.081919 & 7.57 \\
30 & 0.949551 & 0.053130 & 5.04 \\
40 & 0.962198 & 0.039287 & 3.78 \\
50 & 0.969071 & 0.031916 & 3.09 \\
60 & 0.975612 & 0.024997 & 2.44 \\
70 & 0.978597 & 0.021871 & 2.14 \\
80 & 0.980951 & 0.019419 & 1.90 \\
90 & 0.982324 & 0.017994 & 1.77 \\
100 & 0.984930 & 0.015301 & 1.51 \\
\hline 200 & 0.992485 & 0.007572 & 0.75 \\
300 & 0.994996 & 0.005029 & 0.50 \\
400 & 0.996250 & 0.003764 & 0.38 \\
500 & 0.997002 & 0.003007 & 0.30 \\
600 & 0.997503 & 0.002504 & 0.25 \\
700 & 0.997860 & 0.002144 & 0.21 \\
800 & 0.998128 & 0.001875 & 0.19 \\
900 & 0.998337 & 0.001666 & 0.17 \\
1000 & 0.998503 & 0.001499 & 0.15 \\
\hline 2000 & 0.999253 & 0.000747 & 0.07 \\
3000 & 0.999502 & 0.000498 & 0.05 \\
4000 & 0.999621 & 0.000380 & 0.04 \\
5000 & 0.999696 & 0.000304 & 0.0304 \\
10000 & 0.999846 & 0.000154 & 0.0154 \\
\hline 20000 & 0.999923 & 0.000077 & 0.0077 \\
30000 & 0.999949 & 0.000051 & 0.0051 \\
40000 & 0.999961 & 0.000039 & 0.0039 \\
50000 & 0.999970 & 0.000030 & 0.0030 \\
60000 & 0.999975 & 0.000025 & 0.0025 \\
70000 & 0.999978 & 0.000022 & 0.0022 \\
80000 & 0.999981 & 0.000019 & 0.0019 \\
90000 & 0.999983 & 0.000017 & 0.0017 \\
100000 & 0.999985 & 0.000015 & 0.0015 \\
\hline & & & \\
\hline & & & \\
\hline 200 & \\
\hline
\end{tabular}

In Table 1 we show the results of the Kerr ray tracing simulations for nearly face-on rings, $i=1^{\circ}$, in terms of numerical values for $g_{\text {core }}, z_{\text {core }}$ and GR effect as a function of distance to a Kerr black hole with $a / M=0.998$. In principle, Table 1 illustrates the die out of GR with increasing radius. However, it is important to note that according to the asymptotical flatness of GR black holes solutions, space-time curvature approaches zero only in the limit $r \rightarrow \infty$ i.e. there is no finite distance at which the gravitational redshift vanishes exactly. Hence, Table 1 could be generally continued ad infinitum. However, observability poses a limit to what is practical since the resolving power of a spectrograph, in the ideal case, constrains the amount of gravitational redshift (parameterized by $z_{\text {core }}$ here) that can be detected. Assuming a spectral resolution of $0.1 \AA$ for $\mathrm{H} \beta$ as is obtainable by instruments like UVES and CRIRES on the VLT, the corresponding critical value of the $g$-factor is $g=0.999979$. In terms of velocity shift, this is $\approx 10 \mathrm{~km} \mathrm{~s}^{-1}$. As seen in Table 1 , this shift occurs at a radius of $\sim 75000 r_{\mathrm{g}}$. Hence, we do not consider radii above $100000 r_{\mathrm{g}}$ in Table 1 . For supermassive black holes of $10^{7}-10^{8} M_{\odot}$ this radius corresponds to $0.05-0.5$ pc, whereas for stellar-mass black holes of $\sim 10 M_{\odot}$ it is $0.01 \mathrm{AU}$.

Applying Eq. (1) an optical HeII emission line with $\lambda_{\mathrm{em}}=$ $4686 \AA$ in the emitter frame is gravitationally redshifted to

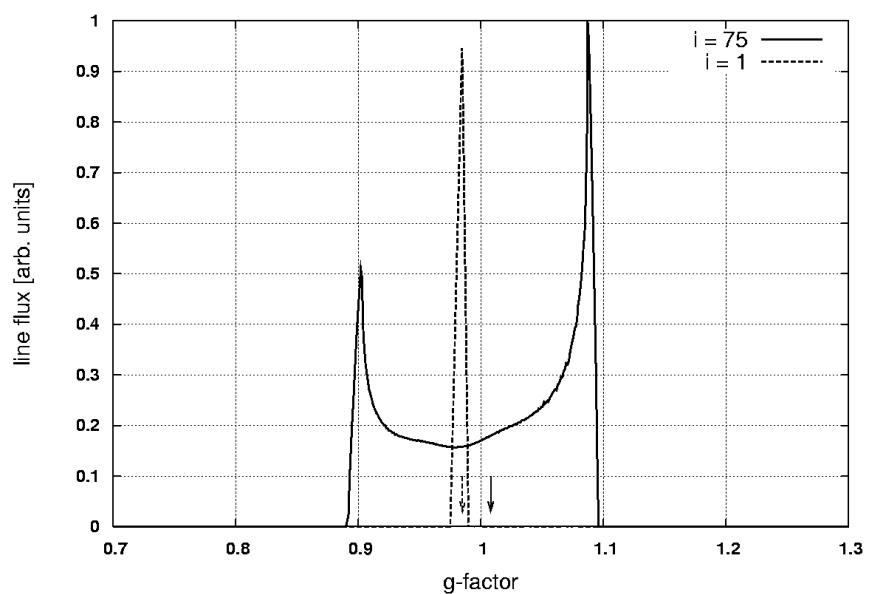

Fig. 3. Two example relativistic lines emitted from a highly inclined ring, $i=75^{\circ}$, and a face-on ring, $i=1^{\circ}$, both with the emission peaking at the same $R_{\text {peak }}=100.0 r_{\mathrm{g}}$ around a Kerr black hole with $a / M=$ 0.998 . The arrows indicate the core $g$-factors, $g_{\text {core }} \simeq 1.008$ and $g_{\text {core }} \simeq$ 0.985 respectively for each particular case.

$4686.1,4686.7,4693.0,4757.7 \AA$ at $100000,10000,1000$, $100 r_{\mathrm{g}}$.

\subsection{Line distortion by strong gravity}

In the previous section the gravitational redshift was investigated as an effect that shifts the whole line core. We now focus on the behaviour of the intrinsic shape of relativistic emission lines as a function of distance to the black hole. This is dubbed strong gravity as anticipated in Sect. 2.2. In the following, we tilt the emitting ring to an inclination of $i=75^{\circ}$ in order to be able to study the characteristic broad profile with the two relic Doppler peaks. Figure 3 shows how the line profile changes by tilting from $i=1^{\circ}$ to $i=75^{\circ}$.

Figure 4 shows the effect of gravitational redshift on the $i=75^{\circ}$ profile as a function of distance to the black hole. The line profile broadens and the line flux gets more and more suppressed as the emitting ring is moved closer to the black hole ${ }^{1}$. Eventually, the line decays and disappears at the event horizon. The red relic Doppler peak is shifted to lower energies as the ring approaches the hole, illustrated in Fig. 5. The red peak flux is also more and more suppressed as can be seen in Fig. 4. Close to the black hole the distortions are so strong that the red Doppler peak becomes highly blurred and effectively vanishes in the line profile. At the event horizon the profile dies out and becomes unobservable. Figure 5 can be used to read the half-energy radius associated with the red relic Doppler peak as defined in Sect. 2.2. At $i=75^{\circ}$ this value is $R_{\text {grp }} \simeq 5 r_{\mathrm{g}}$ and documents that strong gravity is important only very close to the black hole. The distortion of the intrinsic line profile by gravitational redshift can be seen from $g_{\mathrm{rp}}$ alone or by using another line criterion. A further line characteristic is the Doppler peak spacing (DPS) i.e. the energetic distance of both Doppler peaks (if available). DPS can be measured in units of energy, frequency, wavelength or - as has been done here for generality - in units of $g$. Figure 6 shows that this quantity does not remain constant as the black hole is

\footnotetext{
${ }^{1}$ However, it must be noted that the line flux also gets suppressed because the effective emitting area decreases for smaller rings that have constant $r_{\text {out }}-r_{\text {in }}$ in any case.
} 


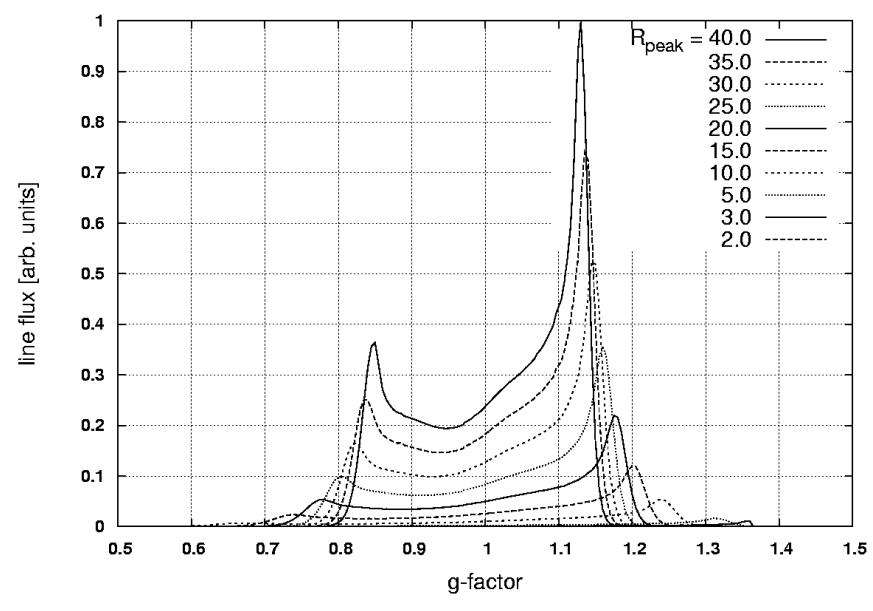

Fig. 4. Line distortion by strong gravity. The emission of narrow rings peaks at the radii as denoted in the legend. There is no way to confuse the lines because line flux successively decreases with decreasing radius of maximum emission $R_{\text {peak }}$. All rings follow Keplerian rotation around a Kerr black hole with $a / M=0.998$ and all are inclined to an inclination angle of $i=75^{\circ}$.

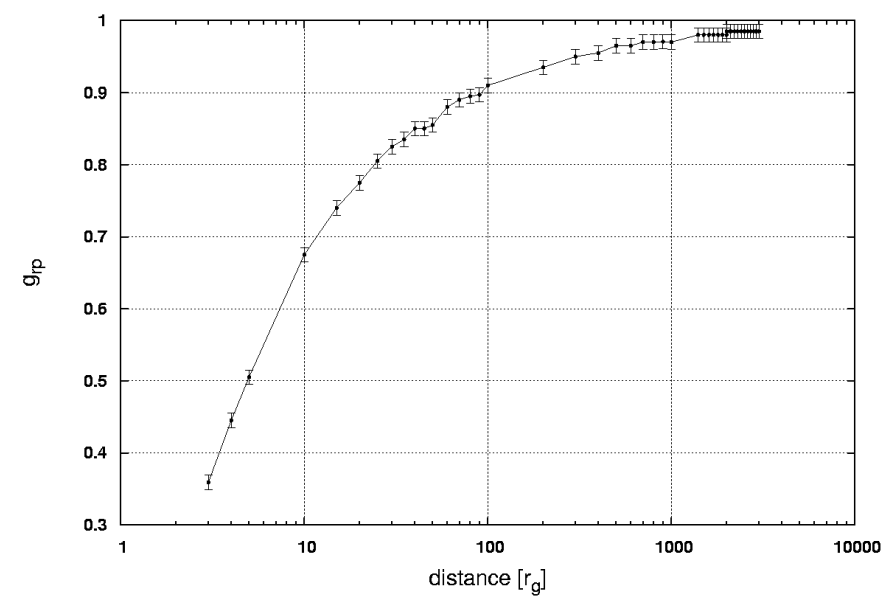

Fig. 5. Distortion of the red relic Doppler peak for rings satisfying $i=75^{\circ}$ : with decreasing radius the red peak can be found at lower peak energies due to strong gravitational redshift. Very close to the black hole $-r \lesssim 3 r_{\mathrm{g}}$ at this specific inclination - these distortions are such strong that the red Doppler peak is highly blurred and vanishes effectively in the line profile.

approached. DPS rises quickly so that the line is stretched. This phenomenon is directly but only qualitatively visible in Fig. 4. Gravitational redshift causes an additional suppression in flux so that close to the black hole the emission line from an intermediately to highly inclined ring is asymmetric and skewed.

We close this section with a comment on black hole detectability: redshifted line cores as presented in Sect. 3.1 leave enough room for other gravitational sources than black holes; in contrast, spectral lines distorted by strong gravity in connection with a measured high compact mass support black hole candidates.

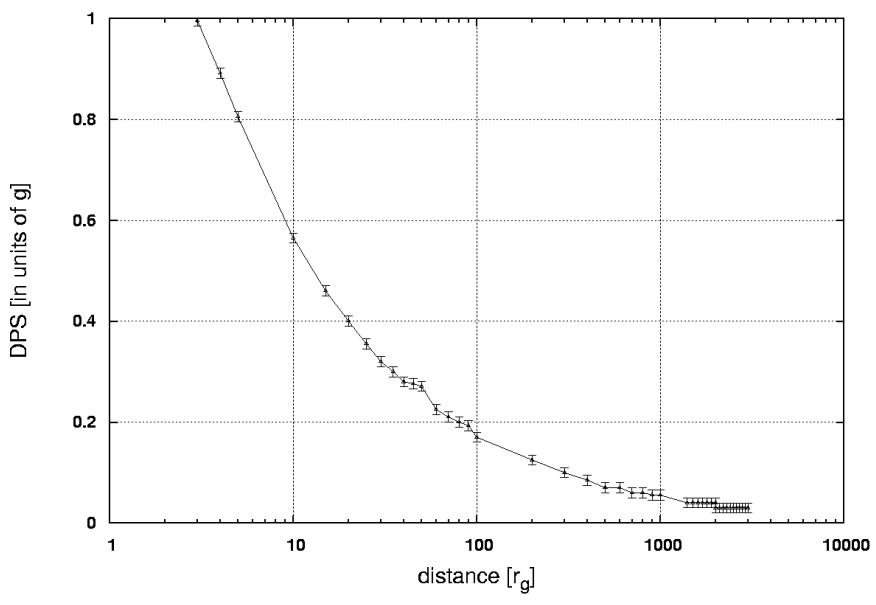

Fig. 6. Energetic distance of red and blue Doppler peak at $i=75^{\circ}$. This Doppler peak spacing (DPS) is measured in units of $g$. Far away from the black hole both peaks approach significantly and the double-peaked line profile becomes very narrow: At $\sim 3000 r_{\mathrm{g}}$ the peak difference in $g$ only amounts to $\sim 0.03$.

\section{Gravitationally redshifted optical emission lines}

\subsection{Observations of NLS-1 Mrk 110}

In a recent work by Kollatschny (2003), broad optical emission lines from $\mathrm{H} \alpha, \mathrm{H} \beta, \mathrm{HeI} \lambda 5876$ and $\mathrm{HeII} \lambda 4686$ were found to display significant and systematic redshifts. By reverberation mapping, the distances of the emitting regions from the central continuum source were determined and a stratified ionization structure seen with HeII arising closest to the black hole at a distance of $\simeq 490 r_{\mathrm{g}}$. The observed shift of the HeII line was measured to be $\Delta z \simeq 0.002$, corresponding to $g \simeq 0.998$.

\subsection{Inner disk inclination of Mrk 110 from Kerr ray tracing}

In this section, we follow the assumption that the observed optical lines of Mrk 110 are subject of gravitational redshift and Doppler shifts and compare them with Kerr ray tracing techniques. It is aimed to determine parameters of the black holeBLR system. The flat BLR model assuming line emitting rings as outlined in Sect. 2.3 is applied. Line redshifts are computed with the fully generalized GR Doppler factors $g$ that were presented by M04 (see Eq. (13) therein). We note that in order to capture the correct $g_{\text {core }}$ value, a fine line binning is needed and hence a very high numerical spectral resolutions is used, $\Delta g=0.00001$.

In contrast to our redshift analysis we now have to allow for arbitrary inclination angles for the BLR in Mrk 110. A parameter space with inclination between $1^{\circ}$ and $40^{\circ}$ and radii in the range 100 and $10000 r_{\mathrm{g}}$ suffices to cover the observational data. The simulated core redshift values follow a power law, $z=p r^{s}$, for fixed inclinations and are plotted in Fig. 7. Observational data for Mrk 110 by K03 are overplotted as boxes. The horizontal error bars are due to the uncertainty in time lag measurements and the vertical error bars involve the uncertainties in the differential redshifts of the rms line centers. The labels of the axes are adapted to theoretical considerations and display redshift $z$ as a function of distance to the black hole in units of gravitational radii. To rescale the $x$-axis the best-fit value for the black hole 


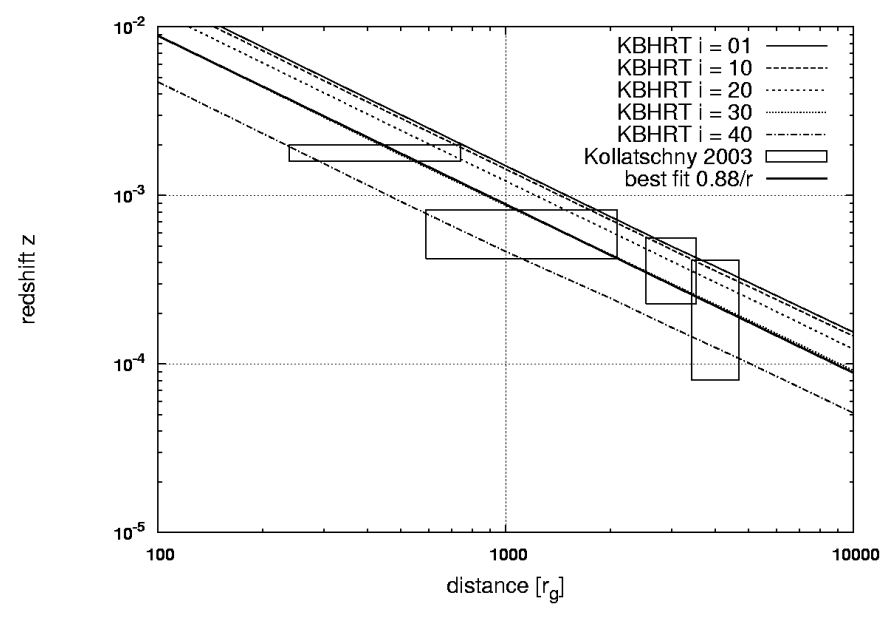

Fig. 7. Radial dependence of the line core redshift: data for the NLS-1 Mrk 110 taken from K03 (boxes) are compared to the line core redshifts as computed from Kerr ray tracing simulations with different inclination angles. Redshift as a function of distance scales with a power law with identical slope $s=-1$ for all inclinations but with different projection parameter $p$ that determines the vertical shift of the power law. Best fit for Mrk 110 (solid thick) yields $p \simeq 0.886$ that can be used to determine the inclination of the inner disk.

Table 2. Power law fit parameters $p$ and $s$ for inclinations $1^{\circ}$ to $40^{\circ}$.

\begin{tabular}{ccccc}
\hline \hline$i\left[^{\circ}\right]$ & $p$ & Error $\Delta p$ & $s$ & Error $\Delta s$ \\
\hline 1 & 1.566 & \pm 0.007 & -1.001 & \pm 0.001 \\
10 & 1.428 & \pm 0.008 & -0.999 & \pm 0.001 \\
20 & 1.187 & \pm 0.003 & -0.996 & \pm 0.001 \\
30 & 0.914 & \pm 0.013 & -1.007 & \pm 0.003 \\
40 & 0.484 & \pm 0.013 & -1.006 & \pm 0.005 \\
\hline
\end{tabular}

mass $^{2}$ obtained by $\mathrm{K} 03$ is assumed, $M \simeq 1.4 \times 10^{8} M_{\odot}$. Table 2 shows the fitting results for power laws at each inclination angle. At any inclination the power law exhibits the same slope, $s_{\text {num }} \approx-1.002 \pm 0.005$, which is the average of the five inclination angles assumed here. This is a direct consequence of the Schwarzschild factor, $z(r) \propto r^{-1}$ i.e. $s_{\text {theo }}=-1$. Additionally, the power laws shift toward lower gravitational redshifts as the inclination angle increases. This is due to projection effects included in the projection parameter $p$. The inclination dependence of the redshift can be approximated by $z \propto \cos (i)$ i.e. $p$ is related to the cosine of $i$. In other words, the higher the inclination, the more blueshift there will be (see Fig. 2), and this Doppler blueshift counteracts the redshift. Interestingly, this behaviour could be exploited to determine the inclination angle of the inner disk from observed gravitationally redshifted features.

The best-fitting power law is shown as a thick solid line in Fig. 7. Here, the slope of $s=-1$ has been fixed and the projection parameter $p$ fitted to give $p_{\text {Mrk } 110} \simeq 0.886 \pm 0.034$. Its relation to a specific inclination $i$ can be extracted from Fig. 8 which shows the cosine behaviour of $p$ for the simulated sets $i \in\left\{1,10,20,30,40^{\circ}\right\}$. The cosine fit to ray tracing data yields $p(i) \simeq 4.63 \cos (i)-3.13$ (error $\sim 10 \%$; note that this is only valid for $i<40^{\circ}$ ) within the radial range between 100 and $10000 r_{\mathrm{g}}$. Taking the best fit value 0.886 one reads in Fig. 8 at the cross that the inner disk of Mrk 110 is inclined to $i \simeq 30^{\circ}$. This result is consistent with that of $\mathrm{K} 03\left(i \simeq 21 \pm 5^{\circ}\right)$.

\footnotetext{
${ }^{2}$ In general, it is recommended to treat $M$ as free parameter, too.
}

\subsection{Static vs. stationary emitter velocity field}

The redshifts calculated above were based on a simple, but dynamical BLR model. In this section we clarify under which conditions the Schwarzschild factor can be used to estimate gravitational redshifts. Usually, a velocity field of the BLR emitters has to be assumed. The prominent Schwarzschild factor can only be applied to quantify the redshift in case of static emitters (and static black holes). Of course, real emitters such as the BLR are dynamical e.g. in stationary motion. In this case, the ray tracing technique is a convenient method for redshift computations. This approach is also justified by the fact that it takes into account that real emitters are extended. The relativistic $g$-factor (consult M04 for details) for static emitters simplifies significantly because the Keplerian angular velocity satisfies $\Omega_{\mathrm{K}}=0$ for radii larger than the radius of marginal stability, $r>r_{\mathrm{ms}}$. The emitter velocity field in Bardeen Observer's frame is assumed to be purely rotational i.e. $v^{(i)}=0$ with $i=r, \theta$. We are thus left with a Lorentz factor $\gamma=\left(1-v_{(\Phi)} v^{(\Phi)}\right)^{-1 / 2}$. Considering $\Omega=\Omega_{\mathrm{K}}=0$ in $v^{(\Phi)}=\tilde{\omega}\left(\frac{\Omega-\omega}{\alpha}\right)$ the Lorentz factor simply goes to unity, $\gamma \rightarrow 1$. It should be kept in mind that this regime is not applicable at $r<r_{\mathrm{ms}}$ when frame-dragging $\omega$ becomes important and $\Omega=0$ is replaced by $\Omega \rightarrow \omega$. Altogether, the generally complicated $g$-factor is identical to the lapse function $\alpha$ for static emitters in the $r>r_{\mathrm{ms}}$ regime. The quantity $\alpha$ is evaluated here for static black holes (as already shown in K03) and for rotating black holes. Restriction to the equatorial plane, $\theta=\pi / 2$, yields a simple expression for the lapse function (see e.g. M04, Eq. (3)):

$\alpha=\sqrt{\frac{r\left(r^{2}-2 M r+a^{2}\right)}{r^{3}+a^{2}(r+2 M)}}$.

The lapse function reduces to the well-known Schwarzschild factor for $a=0$

$\left.\alpha\right|_{a=0}=\sqrt{1-\frac{2 M}{r}}$.

\subsection{Black hole rotation}

A rotating SMBH can not be excluded a priori in the case of Mrk 110. On the contrary, theories of black hole growth, see e.g. Shapiro (2005), strongly suggest fast spinning SMBHs in the local universe with either $a / M \simeq 0.95$ (MHD disk) or even $a / M \simeq 1$ (standard thin gas disk). However, it is also known that the rotation of space-time decays very rapidly in the outer regions of the black hole gravitational potential. The framedragging frequency decreases according to $\omega \propto r^{-3}$. This is documented in the final Fig. 9 which summarizes the results from Figs. 7 and 8 and also extends beyond the region explored by K03. The redshifts corresponding to $g$-factors of $g \longmapsto \alpha$ are plotted for a non-rotating and a fast rotating black hole with $a=0$ (solid curve) and $a / M=0.998$ (dotted curve), respectively. The curves are compared to the observations of Mrk 110 (boxes) and the best-fitting $i=30^{\circ}$ Kerr ray tracing simulation (filled circles). It is evident from the figure that the rotation of space-time is important only at small radii, $r \lesssim 4 r_{\mathrm{g}}$. Therefore, black hole rotation can only be probed with spectral features originating in regions very close to the black hole, like the X-ray fluorescence lines of iron. Optical emission lines are not suited for probing black hole rotation in that manner - at least not for AGN. The reason for the small offset between the ray tracing results shown by the filled circles in Fig. 9 and the 


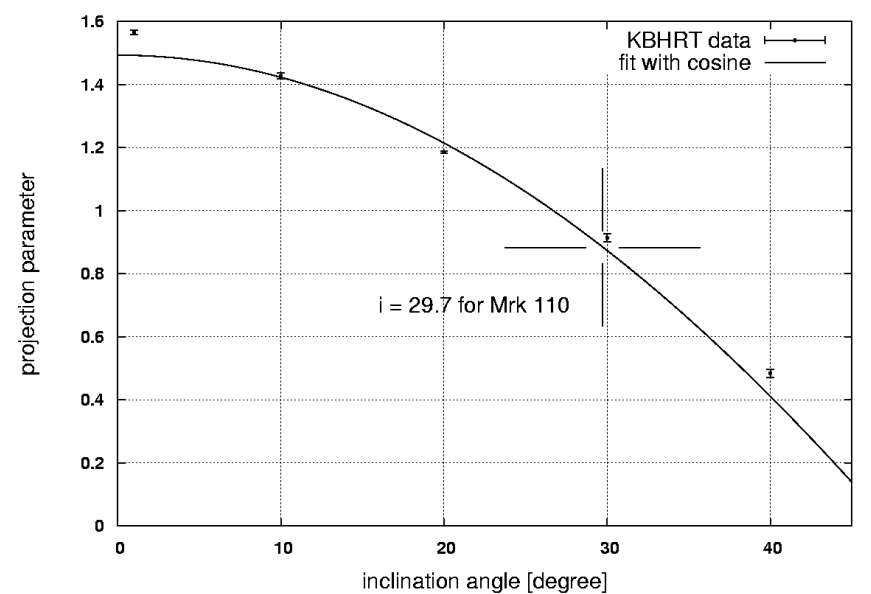

Fig. 8. Variation of the projection parameter $p$ with inclination $i$ as computed from Kerr ray tracing. The numerical data points can be approximated by a cosine function (solid), $p(i) \sim 4.63 \cos (i)-3.13$ within $10 \%$ precision. $p$ measurements from observations give inner disk inclinations. The cross marks the result for Mrk 110 based on K03 data: $i \sim(29.7 \pm 1.2)^{\circ}$.

Schwarzschild/Kerr lapse functions plotted as solid and dotted lines is the different velocity field of the emitters and the transverse Doppler effect. The ray traced emitters are assumed to rotate stationarily, whereas the lapse functions only coincide with ray traced $g$-values when static emitters are assumed. Also the ray traced redshift values are based on $g_{\text {core }}$ computed by averaging over the whole line (Eq. (2)), and therefore are less sharp than those given by the lapse function.

Multi-wavelength observations such as the ongoing COSMOS project ${ }^{3}$ may help filling the gap between 2 and $200 r_{\mathrm{g}}$ in Fig. 9. If several gravitationally redshifted spectral lines can be identified in a source, the central mass can be determined with high accuracy.

\subsection{Assumptions}

BLR emission lines are influenced by both gravitational redshift and Doppler effects. Therefore, the fitting procedure involves two parameters, black hole mass $M$ and inclination angle of the emitter $i$. The inclination can be determined if the black hole mass is known from other methods. In case of Mrk 110, the mass of the SMBH was inferred from reverberation mapping as well as from gravitational redshift in the literature. Other techniques are measurements involving stellar and gas dynamics, the $M-\sigma$ relation and Masers. To be able to determine the inner inclination, we used the best fit black hole mass computed from gravitational redshift found in $\mathrm{K} 03$. The error boxes of $\mathrm{K} 03$ can be fitted quite well with the ray tracing results (3.9\% error). Hence, a model with stationary rotating rings distributed in a radial range between 240 and $4700 r_{\mathrm{g}}$ and inclined to $i \sim 30^{\circ}$ can explain the observed gravitational redshift of optical lines in Mrk 110. It is surprising that a simple Keplerian rotating model can describe the BLR structure so well. Similar results have been obtained e.g. for NGC 5548 (Peterson \& Wandel 1999) and other AGN (Peterson \& Wandel 2000; Onken \& Peterson 2002; Chen et al. 1989). In this work we have made the

\footnotetext{
${ }^{3}$ http://www . astro. caltech.edu/ cosmos/
}

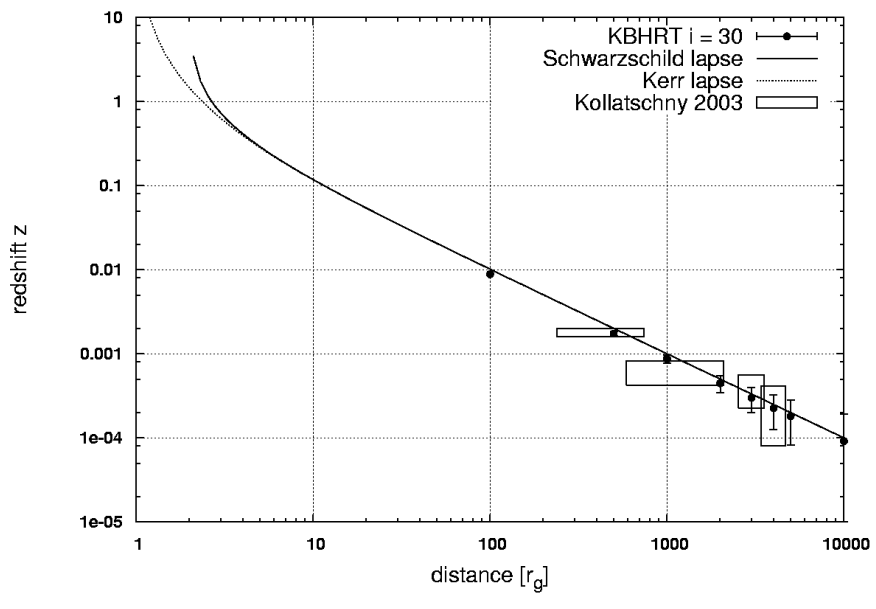

Fig. 9. Synoptical plot with optical K03 data for Mrk 110 (boxes) and best fitting Kerr ray tracing simulation with $i \sim 30^{\circ}$ (filled circles) as well as lapse functions for a static (Schwarzschild, solid) and rotating (Kerr, dotted) black hole. An essential statement illustrated here is that optical BLR lines can not probe black hole rotation due to their huge distance. Generally, multi-wavelength observations are recommended to fill the gap at smaller radii.

assumptions of a flat and Keplerian rotating BLR structure. However, nature is supposed to be more complicated allowing particularly for BLR wind components and opacity effects (Popovic et al. 1995; Chiang \& Murray 1996). This implies a modification of the solver including radial and poloidal motion as well as photon propagation through a dense volume. Furthermore, disk warping has not been considered and this may significantly change the results. For warped disks the analysis has to be extended by using suitable generalized ray tracing codes e.g. like the one by Cadez et al. (2003).

\section{Conclusions}

Line cores at distances from 2 to $100000 r_{\mathrm{g}}$ from a rotating black hole have been analysed using relativistic ray tracing simulations in the Kerr geometry. The line cores are gravitationally redshifted by $z_{\text {core }} \simeq 10^{-5}, 10^{-4}, 10^{-3}, 10^{-2}, 10^{-1} 10^{0}$ at distances of $100000,10000,1000,100,10,2 r_{\mathrm{g}}$ from the black hole, respectively. This $z \approx \frac{1}{\text { distance }\left[r_{g}\right]}$ behaviour at large radii is a straightforward consequence of the Schwarzschild factor. Lines characterised by a core energy $g_{\text {core }}$ confirm this scaling behaviour.

Gravitational redshift occurs in two modes. One regime starts at larger distances from the black hole and shifts only the line as a total feature while conserving its intrinsic shape; the amount of redshift can be looked up in Table 1. The other regime, which is the strong gravity regime, dominates at $r \lesssim 10 r_{\mathrm{g}}$ or at $r \lesssim 10^{13} \mathrm{~cm}$ for a ten million solar mass black hole. Relativistic emission lines originating in this region are strongly deformed and suppressed and differ substantially from the corresponding line profiles in the emitter's rest frame.

The present work has demonstrated that the onset of GR becomes important at distances smaller than $75000 r_{\mathrm{g}}$ if assuming an optical spectral resolution of $0.1 \AA$. It is stressed that this critical radius depends on the astronomical resolving power and lies farther out if the resolution is higher. However, 
even with high-resolution spectroscopy it remains a challenge to probe gravitationally redshifted spectral lines due to the fact that competing effects and more complex physics are likely to be involved: a flat Keplerian BLR model may be too simple to model the complex BLR velocity field and both spherical BLR structure and a wind component may play a crucial role. In addition, narrow-line components and e.g. contamination of the $\mathrm{H} \beta$ line by FeII may also complicate both the analysis and the interpretation. Nevertheless, the theory of General Relativity predicts that the gravitational redshift is present, and a valuable ansatz for probing it is to search for systematic shifts with varying distance, as done by $\mathrm{K} 03$. It is suggested in this work to supplement this by multi-wavelength observations that should all point towards the same central mass and inner inclination.

We confirm the analysis by K03 of the NLS-1 galaxy Mrk 110 here, using a more general treatment with stationary $g$-factors that are in concordance with observational data. Ray tracing simulations in the Kerr geometry support an inclination of $i=30^{\circ}$ for the inner disk of Mrk 110 . Reversely, if the inclination is known, this can be exploited to determine the black hole mass from the fitting procedure outlined here. Whether fitting $i$ or $M$ (or both) - such techniques may help explore AGN unification schemes: multi-wavelength studies allow for studying the inclination deep into the AGN and to probe orientation and luminosity-dependence of AGN types. Furthermore, we show that broad optical lines can not serve as a probe of black hole spin because frame-dragging effects only occur very close to the black hole. In this region, only hot emission lines (such as the Fe $\mathrm{K} \alpha$ line in X-rays) or other relativistic spectral features indicate black hole spin. The analysis presented here is not only valid for supermassive black holes but also for stellar-mass black holes in X-ray binaries or intermediate-mass black holes that may be found in ultra-luminous X-ray sources or globular clusters.
Acknowledgements. A.M. wishes to thank the organizers and participants of the Japanese-German meeting in Wildbad Kreuth, Germany, especially Wolfram Kollatschny (University of Göttingen) and Lutz Wisotzki (AIP). We wish to thank John Silverman (MPE) for inspiring discussions.

\section{References}

Blandford, R. D., \& McKee, C. F. 1982, ApJ, 255, 419

Cadez, A., Brajnik, M., Gomboc, A., Calvani, M., \& Fanton, C. 2003, A\&A, 403, 29

Chen, K., Halpern, J. P., \& Filippenko, A. V. 1989, ApJ, 339, 742

Chiang, J., \& Murray, N. 1996, ApJ, 466, 704

Collin, S., Kawaguchi, T., Peterson, B., \& Vestergaard, M. 2006, ArXiv Astrophysics e-prints

Corbin, M. R. 1997, ApJ, 485, 517

Cunningham, C. T. 1975, ApJ, 202, 788

Czerny, B., Rózańska, A., \& Kuraszkiewicz, J. 2004, A\&A, 428, 39

Fabian, A. C., Rees, M. J., Stella, L., \& White, N. E. 1989, MNRAS, 238, 729

Kaspi, S., Smith, P. S., Netzer, H., et al. 2000, ApJ, 533, 631

Kollatschny, W. 2003, A\&A, 412, L61

Konigl, A., \& Kartje, J. F. 1994, ApJ, 434, 446

Lynden-Bell, D. 1969, Nature, 223, 690

Lynden-Bell, D., \& Rees, M. J. 1971, MNRAS, 152, 461

Müller, A., \& Camenzind, M. 2004, A\&A, 413, 861

Netzer, H. 1977, MNRAS, 181, 89P

Netzer, H. 2003, ApJ, 583, L5

Onken, C. A., \& Peterson, B. M. 2002, ApJ, 572, 746

Peterson, B. M. 1993, PASP, 105, 247

Peterson, B. M., \& Wandel, A. 1999, ApJ, 521, L95

Peterson, B. M., \& Wandel, A. 2000, ApJ, 540, L13

Peterson, B. M., Meyers, K. A., Carpriotti, E. R., et al. 1985, ApJ, 292, 164

Peterson, B. M., Berlind, P., Bertram, R., et al. 2002, ApJ, 581, 197

Popovic, L. C., Vince, I., Atanackovic-Vukmanovic, O., \& Kubicela, A. 1995, A\&A, 293, 309

Robinson, A., Perez, E., \& Binette, L. 1990, MNRAS, 246, 349

Ruiz, J. R., Crenshaw, D. M., Kraemer, S. B., et al. 2001, AJ, 122, 2961

Shapiro, S. L. 2005, ApJ, 620, 59

Tanaka, Y., Nandra, K., Fabian, A. C., et al. 1995, Nature, 375, 659

Thorne, K. S. 1974, ApJ, 191, 507

Woltjer, L. 1959, ApJ, 130, 38

Zheng, W., \& Sulentic, J. W. 1990, ApJ, 350, 512 